\title{
BMJ Open Mutaba'ah-Mother and Child Health Study: protocol for a prospective cohort study investigating the maternal and early life determinants of infant, child, adolescent and maternal health in the United Arab Emirates
}

\author{
Amal Al Haddad, ${ }^{1}$ Nasloon Ali, ${ }^{1}$ Iffat Elbarazi, ${ }^{1}$ Haba Elabadlah, ${ }^{2}$ \\ Fatima Al-Maskari, ${ }^{1,3}$ Hassib Narchi, ${ }^{\circ}$ Christel Brabon, ${ }^{5}$ Saad Ghazal-Aswad, ${ }^{6}$ \\ Fatima M AIShalabi, ${ }^{7}$ Antonis Zampelas, ${ }^{8}$ Tom Loney, ${ }^{1,9}$ lain Blair, ${ }^{1}$ Luai A Ahmed $^{\oplus 1}$
}

To cite: Al Haddad A, Ali N, Elbarazi I, et al. Mutaba'ahMother and Child Health Study: protocol for a prospective cohort study investigating the materna and early life determinants of infant, child, adolescent and maternal health in the United Arab Emirates. BMJ Open 2019;9:e030937. doi:10.1136/ bmjopen-2019-030937

- Prepublication history and additional material for this paper are available online. To view these files, please visit the journal online (http://dx.doi. org/10.1136/bmjopen-2019030937).

Received 08 April 2019 Revised 17 July 2019 Accepted 19 July 2019

Check for updates

(c) Author(s) (or their employer(s)) 2019. Re-use permitted under CC BY-NC. No commercial re-use. See rights and permissions. Published by BMJ.

For numbered affiliations see end of article.

Correspondence to

Dr Luai A Ahmed;

luai.ahmed@uaeu.ac.ae

\section{ABSTRACT}

Introduction Early life exposures, particularly

environmental and parental lifestyle factors, have a major influence on children's health and development. Due to increasing interest in the early life developmental origins of diseases, many birth cohorts have been established. These studies constitute a repository of data which researchers use over many years to investigate emerging research questions. However, no such databank or cohort study is available in the United Arab Emirates (UAE). This project aims to establish a prospective mother and child cohort study in Al Ain (Abu Dhabi, UAE) to investigate the maternal and early life determinants of infant, child, adolescent and maternal health of the Emirati population.

Methods and analysis During the period 2017-2021, this study aims to recruit 10000 pregnancies at approximately 12 weeks of gestation from hospitals and clinics in Al Ain city. For each mother/newborn pair, an initial dataset will be collected including anthropometric, physiological and biochemical measurements, medical interventions, circumstances of pregnancy, delivery details and neonatal and perinatal growth and health using a combination of questionnaires, interviews and medical record extractions. Baseline data will act as the starting point from which the children will be followed up and re-surveyed at intervals throughout their life course until the age of 16 years, to explore how familial, socioeconomic and lifestyle factors interact with genetic and environmental factors to influence health outcomes and achievements later in life.

Ethics and dissemination Ethical approval has been granted by the United Arab Emirates University Human Research Ethics Committee and the ethical committees of the participating institutions. Results will be widely disseminated via peer-reviewed manuscripts, conference presentations, media outlets and reports to relevant authorities.

\section{Strengths and limitations of this study}

- This study is the largest mother and child cohort study to be established in the United Arab Emirates, and the first conducted in the emirate of Abu Dhabi.

- The study will have a large sample size of 10000 pregnancies as well as a long follow-up period (16 years).

- We intend to recruit pregnant women from the Emirati population who would be more likely to follow-up till the Emirati children are 16 years of age.

- Standardised scales and clinical assessments and records from hospitals are carefully designed to reduce any misreporting and ensure a large cohesive dataset.

- We are not recruiting other expatriates as it might lead to a large amount of attrition, so the study may not be generalisable to populations elsewhere.

\section{INTRODUCTION}

Although the causes of non-communicable disease epidemics have not yet been fully determined, emerging evidence suggests causal associations between their occurrence and exposures during pregnancy and early life. ${ }^{12}$ The Developmental Origins of Health and Disease hypothesis speculates that the risk of chronic disease in adult life is related to biological programming of the fetus or infant in response to early environmental signals. ${ }^{3}$ Moreover, there is convincing evidence that physical and psychosocial exposures in the first months of life and later in childhood have important effects on health, well-being and development during adolescence and adulthood. ${ }^{4-6}$ For instance, numerous studies have reported the beneficial effect of breast 
feeding on some of the major components of the metabolic syndrome (ie, obesity, blood pressure, cholesterol metabolism and insulin resistance) and thereafter on the risk of cardiovascular disease. ${ }^{78}$

Research based on the available medical record systems or on cross-sectional data usually involve small samples of the general population with limited information on specific exposures and disease outcomes of interest. First, this will significantly narrow exposure-outcome relationships. Second, these studies will face methodological and analytical problems of residual confounding due to the lack of data on other important lifestyle, socioeconomic, nutritional, physical and environmental exposures not routinely captured in medical records. Therefore, new datasets that support research in a life course perspective are urgently needed. Datasets with rich information on exposures and outcomes, which include social and biological factors, can be used by researchers in different fields with widely different types of causal models to produce original research. For over half a century, there has been a proliferation of birth cohort studies fueled by the recognition of the importance of the in utero environment on health outcomes in later life. ${ }^{9}$ The main distinctive feature of a cohort study is the longitudinal follow-up time frame and the frequency of the follow-up that could be extended indefinitely. ${ }^{4}$ A longitudinal design allows the quantification of the temporal relationship between a specific exposure and outcome, observance of the natural history and calculation of the incidence of a specific outcome. Despite the surge in the number of cohort studies, there is a lack of longitudinal studies in the United Arab Emirates (UAE) with follow-ups across the lifespan to help understand the aetiology of numerous health conditions in the community.

This protocol describes the research design and methods used to establish the Mutaba'ah study, which is a large prospective cohort study focusing on maternal and child health in the UAE. This study aims to establish a prospective mother and child cohort study in Al Ain city to investigate the maternal, genetic, social, environmental, lifestyle and other early childhood determinants of infant, child and adolescent health, as well as the mother's health.

The study objectives are to: (i) identify and collect data on relevant exposures of the mother and child during pregnancy, delivery and the postnatal period; (ii) identify and collect relevant outcomes of the mother and child during delivery, postnatal period and early life; (iii) associate relevant exposures and health outcomes and (iv) establish a prospective and updated databank.

\section{METHODS AND ANALYSIS}

\section{Study design}

Mutaba'ah (meaning 'follow-up' in Arabic), the mother and child health study is a prospective cohort study in $\mathrm{Al}$ Ain city.

\section{Study setting}

Al Ain is the second largest city in the Emirate of Abu Dhabi (the largest UAE emirate in terms of land mass and population size) and has the largest and relatively stable population of Emirati citizens compared with other parts of the country. This makes it an ideal setting for a birth cohort study with longitudinal follow-ups. In 2016, the Emirate of Abu Dhabi population was estimated to be approximately 2.9 million, of which, $<19 \%$ were Emiratis and around $41 \%$ of these Emiratis lived in the $\mathrm{Al}$ Ain region. ${ }^{10}$ The population of $\mathrm{Al}$ Ain is approximately 766 900 (mid-2016) of which 226300 are Emirati citizens and of these 53995 are women of reproductive age (15-44 years).${ }^{10}$ Levels and standards of antenatal care are high, and all births take place in hospitals. Although this study has the potential to expand to involve many health institutions, the initial recruitment has been confined to three major hospitals in $\mathrm{Al} \mathrm{Ain:} \mathrm{the} \mathrm{only} \mathrm{two} \mathrm{public}$ hospitals and one large private hospital; Al Ain Hospital, Tawam Hospital and Oasis Hospital, respectively. As all of the Emirati population has full health insurance allowing them to have the same level of healthcare at any health facility, there is no difference in healthcare access between pregnant women attending these three hospitals and those who use other institutions. Therefore, a representative sample of the Emirati population in $\mathrm{Al}$ Ain can be recruited from these three hospitals.

\section{Participants}

The study aims to recruit 10000 mother and baby pairs with each pair suggesting a pregnancy.

\section{Inclusion criteria}

Invitation for participation is limited to the Emirati population. All pregnant women from this population who are attending any of the three participating hospitals for their antenatal care management and who are at least 18 years of age, resident in $\mathrm{Al}$ Ain, ideally in their first trimester (approximately 12 weeks of gestation), and are able to provide informed consent, as well as their newborns will be included in the study. Women with multiple pregnancies (pregnant with more than one fetus) and those who conceive multiple times during the study period, as well as their offspring will also be included in the study so long as they provide consent.

\section{Exclusion criteria}

The study will exclude expatriates as they are less likely than the Emirati population to be available for long-term follow-up. Pregnant women younger than 18 years or those who are unable to provide consent and women who are not currently pregnant, will also be excluded.

\section{Sampling and recruitment}

There are approximately 6600 births of Emirati children each year in $\mathrm{Al} \mathrm{Ain,} \mathrm{of} \mathrm{which,} \mathrm{the} \mathrm{clear} \mathrm{majority} \mathrm{take} \mathrm{place}$ in the three participating hospitals. The study employs a consecutive sampling strategy whereby all eligible pregnant women that present at one of three hospitals are 
invited to participate in the study. Eligible participants are identified via a healthcare provider (nurse or physician) at each hospital's registration point and are approached by an on-site research assistant with an information sheet detailing the project. If they express interest, participants provide informed consent. This consent allows for follow-up interviews and the extraction of their and their babies' health information from medical records up until the child is 16 years of age. The study is conducted in accordance with the principles of the Declaration of Helsinki. Each participant has the right to withdraw from the study at any time without giving any reason. During enrolment, the participant is provided with a detailed information sheet on the Mutaba'ah study description, contact details (telephone number and email address) and the withdrawal process. In addition, the withdrawal process is explained verbally to the participant. The participant is informed verbally and in writing that they can call or email at any time to execute their right to withdraw from the study. Should a participant wish to withdraw from the study, and wish for their collected data to be destroyed, this will be completed in a prompt and secure manner and a message will be sent to the woman to confirm study withdrawal. The reason for withdrawal, if given, will be recorded in the database.

\section{Patient involvement}

Participants provide input during their recruitment process on their thoughts on how best to follow them and their children up after delivery, and how to access more participants. This information is usually collected verbatim by the data collectors or collected via administrative forms. Participants were not consulted about study outcomes or interpretation of the results.

\section{Data sources and measurements}

Collected data will include but are not limited to: demographic, socioeconomic, lifestyle, environmental, education, employment, physical and mental health, household and family information, parental health, social support, local community and services, mother and child nutrition, previous pregnancies and birth outcomes, health and development of child and childcare information.

Data will be collected around the following time points: 12 weeks of gestation; 25 weeks of gestation; at the time of delivery and at 6 and 12 months for the infants. The ideal timeline of data collection is shown in figure 1. Further time points for follow-up during childhood and adolescence will be decided later. In addition to data abstracted from the medical records, data will be obtained using three tablet-assisted self-administered questionnaires: a short questionnaire (SQ), long questionnaire (LQ) and a food frequency questionnaire (FFQ). The research database will be linked using the medical record number and unique identification number assigned to each participant when consenting to the study. Medical records of the mother will provide information on the progress of the pregnancy and pregnancy outcomes. Information

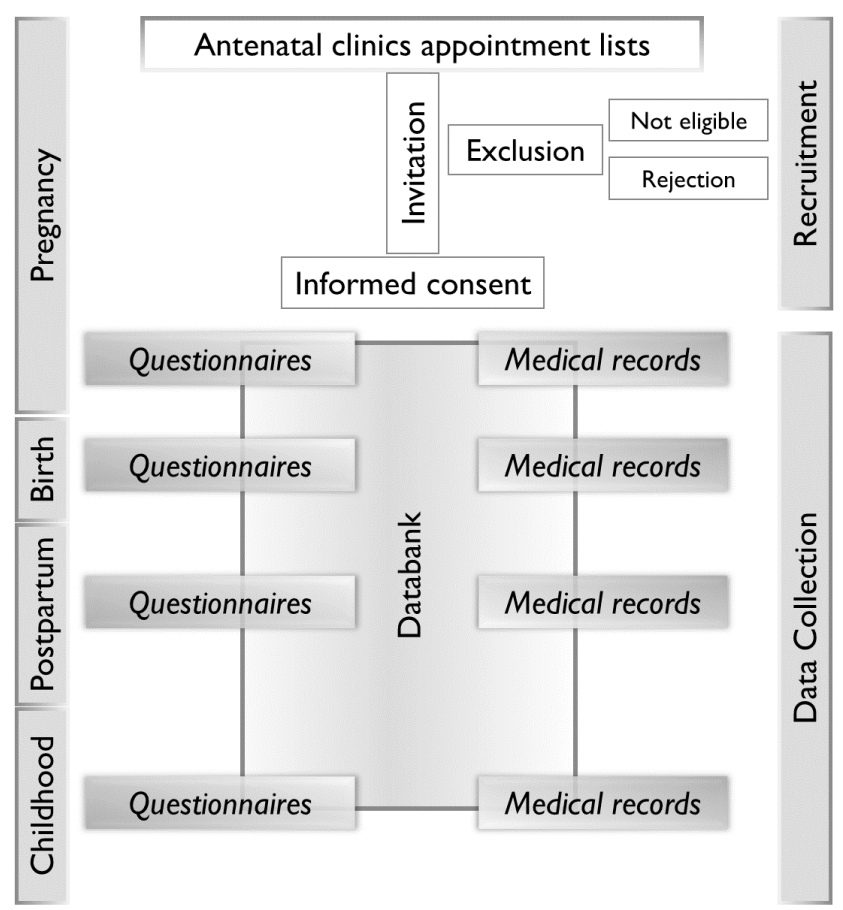

Figure 1 Data collection time points during the Mutaba'ah study.

will be extracted from the laboratory tests and imaging results which are performed routinely on all women. These routine antenatal care screenings and tests include among others, complete blood count, vitamin D (plasma 25-hydroxyvitamin D3), infectious disease screening (ie, hepatitis B virus, HIV, rubella, syphilis, varicella), cervical cancer screening, haemoglobinopathy screening, anomaly scan, gestational diabetes mellitus screening and repeated measures of proteinuria, fetal growth, heart tones, fundal height measurements, maternal body mass, body mass index and blood pressure. All medical outcomes will be ascertained from the woman's and child's medical records as standardised definitions are employed following the regulations and guidelines of the Department of Health of Abu Dhabi. ${ }^{11}$ Official birth notifications and delivery records will provide data on the delivery and birth outcomes. Important information on genetic exposures and outcomes will be collected from the medical records and questionnaires. Examples of genetic data include degree of consanguinity, family history of diseases and routine genetic screening results of the mother and child such as thalassaemia, glucose6-phosphate dehydrogenase deficiency and sickle cell trait. Interviews using the infant questionnaire will be conducted via follow-up hospital visits, home visits or telephone when the child is 6 and 12 months of age. In the future, we plan to collect data when the child is aged from 5 to 16 years using interviews or specifically designed child questionnaires and adolescent questionnaires. We plan to collaborate with federal institutions to set-up linkages between the Mutaba'ah study data and UAE population registries to ensure continued access to the child's 
Table 1 Examples of data collected at different time points in the study

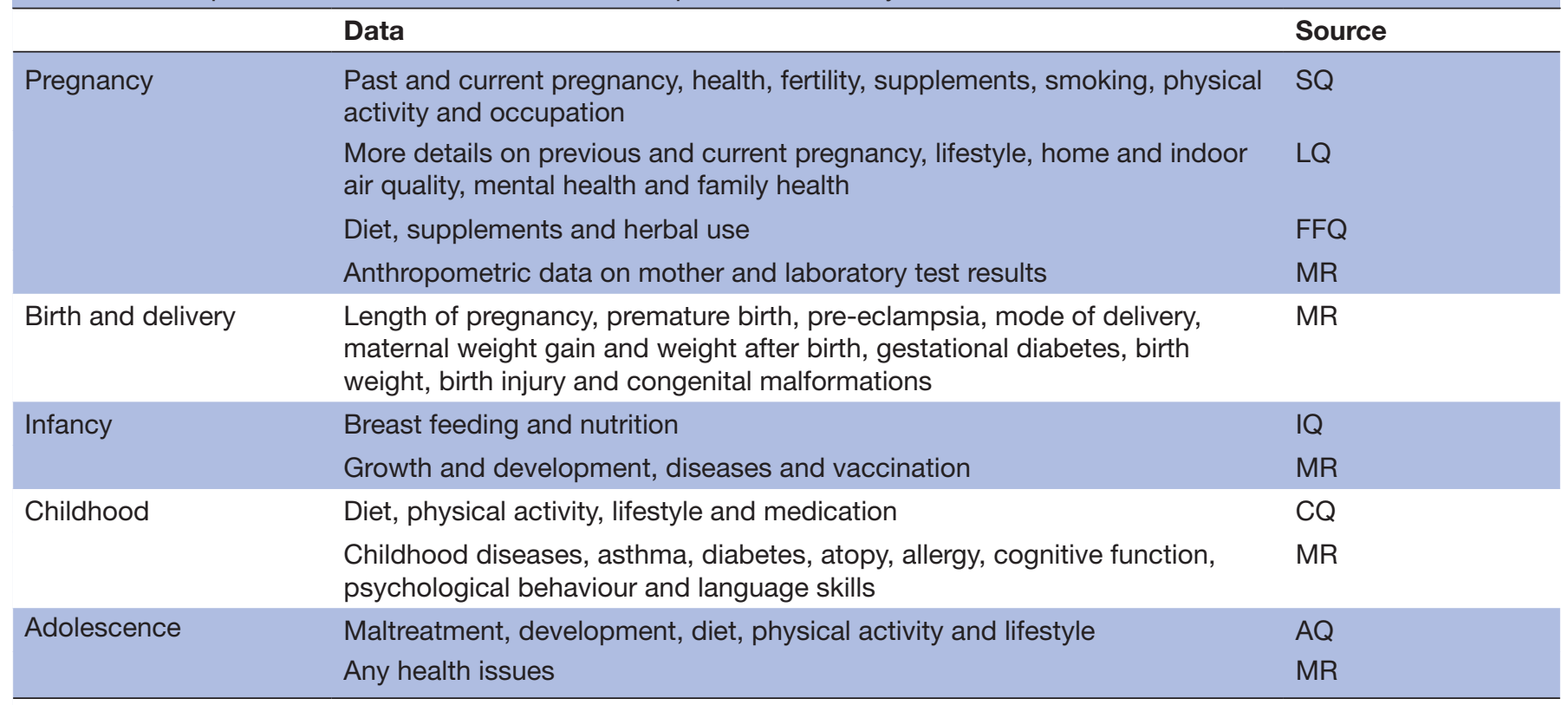

$A Q$, adolescent questionnaire; $C Q$, child questionnaire; $F F Q$, food frequency questionnaire; IQ, infant questionnaire; $L Q$, long questionnaire; MR, medical records; SQ, short questionnaire.

health and education records during their childhood and adolescence.

Table 1 shows examples of the types and sources of data collected during the study. The SQ is administered at the first point of contact and comprises 67 questions on varying sociodemographic and psychosocial measures. Questions were pooled and adapted from the Avon Longitudinal Study of Parents and Children, ${ }^{12}$ the Norwegian Mother and Child Study, ${ }^{13} 14$ the Danish Birth Cohort Study ${ }^{15}$ and the Born in Bradford study. ${ }^{16}$ The semi-quantitative FFQ will be completed by the mother during the second or third trimester to ascertain diet (ie, energy intake, macronutrient and micronutrient intake), supplement intake and herbal use during the pregnancy. The FFQ has been created and validated in the UAE and the nutrient intake calculations will be based on a newly developed food composition database developed specifically for the UAE. ${ }^{17}$

The LQ will be a combination of questions asked in the four cohort studies previously mentioned that were used to develop the SQ as well. However, it will delve deeper into psychosocial dimensions of health including mental health using scales such as the Edinburgh Depression Scale $^{18}$ and the Spielberger State Trait Anxiety Inventory. ${ }^{19}$ All questionnaires were translated from English to Arabic by one group of study researchers and then back translated from Arabic to English by a different group of study researchers.

During childhood and adolescence, we will use previously validated measures such as the Childhood Trauma Questionnaire, ${ }^{20}$ Adverse Child Experiences, ${ }^{21}$ a child-relevant $\mathrm{FFQ}^{22}$ and the Physical Activity Questionnaire for
Older Children and Physical Activity Questionnaire for Adolescents. ${ }^{23}$

Clinical data will be abstracted by the research team using a standardised chart abstraction tool to ascertain prenatal and perinatal conditions, anthropometrics of both mother and child, delivery and birth outcomes and childhood diseases. We will not be collecting any biological samples at this stage of the study. The online supplementary table provides more details on the proposed and projected variables, time points and data sources in the Mutaba'ah study.

\section{Sample size}

Assuming an exposure level of $10 \%$ and a relatively common outcome of $5 \%$ (for instance, sickle cell trait ${ }^{24}$ ) in the unexposed group, a cohort of 10000 pregnancies will allow the detection of true relative risks of approximately 0.61 or 1.45 in exposed group relative to unexposed group with $80 \%$ power and a $5 \%$ type I error probability that this relative risk equals 1 . Accounting for a $20 \%$ attrition rate, table 2 shows the minimum detectable relative risks for different levels of exposure and proportions of affected unexposed based on a cohort of 8000 and $80 \%$ power and a $5 \%$ type I error probability.

\section{Statistical methods}

Crude and adjusted prevalence and incidence of key outcomes will be estimated with their 95\% CIs. Descriptive statistics will be performed to show the distribution of the study population characteristics. Continuous variables will be presented as means with SD or medians with IQR where appropriate, while categorical variables will be presented as counts (percentages). Continuous variables 
Table 2 Minimum detectable relative risks for different levels of exposure and proportions of affected unexposed based on a cohort of 8000 with $80 \%$ power and $5 \%$ type I error probability

\begin{tabular}{lllllll}
\hline & & \multicolumn{7}{l}{ Proportion of outcome in unexposed group } & \\
\cline { 4 - 7 } & Exposure level (\%) & $\mathbf{1 \%}$ & $\mathbf{5 \%}$ & $\mathbf{1 0 \%}$ & $\mathbf{2 5 \%}$ & $\mathbf{5 0 \%}$ \\
\hline Protective exposure & $\mathbf{1}$ & - & - & 0.202 & 0.491 & 0.688 \\
& $\mathbf{5}$ & - & 0.425 & 0.588 & 0.753 & 0.853 \\
& $\mathbf{1 0}$ & 0.125 & 0.562 & 0.69 & 0.816 & 0.891 \\
Harmful exposure & $\mathbf{2 5}$ & 0.332 & 0.68 & 0.775 & 0.867 & 0.922 \\
& $\mathbf{5 0}$ & 0.427 & 0.727 & 0.808 & 0.887 & 0.934 \\
& $\mathbf{5 0}$ & 1.76 & 1.307 & 1.207 & 1.117 & 1.066 \\
& $\mathbf{2 5}$ & 1.897 & 1.362 & 1.244 & 1.138 & 1.078 \\
& $\mathbf{1 0}$ & 2.276 & 1.51 & 1.342 & 1.192 & 1.109 \\
& $\mathbf{5}$ & 2.782 & 1.701 & 1.468 & 1.261 & 1.147 \\
& $\mathbf{1}$ & 5.341 & 2.612 & 2.051 & 1.57 & 1.312 \\
\hline
\end{tabular}

with a normal distribution will be compared using a Student's t-test or analysis of variance. Categorical data will be compared using the Pearson's $\chi^{2}$ test. Univariate and multivariate regression models will be used to quantify the association between potential exposures and the different outcomes. Multiple imputation, complete-case analyses and sensitivity analyses will be used to deal with missing data. Logistic and linear models will be used for binary and continuous outcome variables, respectively. Propensity score analysis, survival analyses and proportional hazard models will be used when applicable. Crude and adjusted ORs, relative risks and HRs with 95\% CIs will be reported. Statistical analyses will be performed using STATA and $R$ statistical packages. $P$ value $\leq 0.05$ will define statistical significance.

\section{ETHICS AND DISSEMINATION}

Informed written consent is obtained from the participant prior to the data collection.

It is anticipated that Mutaba'ah will receive widespread interest in the UAE as well as neighbouring regions and media attention. We also anticipate our results to be published in highly ranked international scientific journals, presented at conferences and meetings as well as reported to appropriate authorities for interventions and services.

\section{STATUS OF PROJECT}

Pilot recruitment was performed between May and August 2017 (100 participants), while active recruitment began in October 2017 and will proceed till 2021 or until the desired sample size has been reached. Around 5000 participants completed the SQ (among them 844 participants completed LQ) until May 2019. With the current participation rate of over $80 \%$, it is expected that over 6000 pregnant women could realistically be recruited by the end of second year (October 2019) with the remainder to be recruited in the third year of the study. The refusal rate is around $17.5 \%$. Women who refuse generally indicated either fatigue or lack of time as their reasons for refusal. We have had three withdrawals so far citing personal reasons. Despite a steady enrolment, we face some challenges as most longitudinal cohorts with respect to partial responses and loss to follow-up. The total number of variables in our dataset currently amounts up to $>1800$ variables from the two questionnaires and medical records.

\section{DISCUSSION}

In this paper, we present the design, data collection methods and expected analyses of a mother and child cohort study in Al Ain-the Mutaba'ah study. The study aims to investigate the maternal and early childhood determinants of infant, child and adolescent health, as well as maternal health. The pregnant women recruited from the three participating hospitals are expected to be representative of the local population and will provide a rich depiction of the risk and protective factors experienced by the cohort as a whole.

Currently, there is only one ongoing 2-year prospective birth cohort study (Mother-Infant Study Cohort (MISC)), which recruited 256 of both UAE national and expatriate pregnant women late during their pregnancy in three emirates (Dubai, Sharjah and Ajman) of the UAE. ${ }^{25}$ The 2-year follow-up period in the MISC study focuses on exploring the association between nutrition and lifestyle characteristics on birth outcomes, infant nutritional status and cognitive development. Mutaba'ah on the other hand aims to recruit pregnant women during the first trimester of their pregnancy to enable exploring the early pregnancy exposures that influence maternal and fetal health during the second and third trimester, in addition to delivery complications and birth outcomes. Moreover, the planned long-term follow-up (16 years) will permit Mutaba'ah to investigate the prenatal and neonatal exposures that might be associated with infant, child and adolescent health outcomes. Both the MISC 
and Mutaba'ah studies will enrich the scant body of maternal and child health research in the UAE.

The strengths of the Mutaba'ah study include the establishment of the largest longitudinal cohort of pregnant women and their children in the UAE. The prospective population-based design with the planned long follow-up of a large cohort of mothers and children has great advantages. The study will be able to collect high-quality clinical data from medical records which will reduce missing data and imprecision in variables such as body mass and gestational age. Therefore, Mutaba'ah will secure epidemiological data and provide a pioneering platform for maternal and child health research. As in most epidemiological studies, self-reported data and loss to follow-up (at least beyond birth) are potential weaknesses to this study.

Exposures to various risk factors in utero and during childhood have a major influence on health and development in adulthood. The impact of this study will extend beyond exposing factors that will be risky and protective for delivery and birth outcomes, childhood and adolescent health. Mutaba'ah will create a databank for likeminded researchers to conduct work on maternal and child health in the country. It will provide insights into unique exposures and outcomes present in the region such as consanguinity, ${ }^{26}$ increasing rates of obesity in women and children, ${ }^{27} 28$ gestational diabetes ${ }^{29}$ and smoking practices such as shisha. ${ }^{30}$ The study will also provide opportunities to use case-cohort and nested case-control designs for some unique research questions and to perform validation studies. Mutaba'ah will aim to initiate regional and international collaborations with similar ongoing mother and child cohort studies to enable performing international comparisons and generating more research prospects.

Cohort studies with such breadth and depth of data can provide important information to the public and authorities. This study will allow researchers to relate different health outcomes to a variety of early life exposures using appropriate statistical methods based on the robust longitudinal cohort design. Study findings will provide the local, regional and international scientific community with evidence of the burden and impact of diseases in maternal and child health in a high-income Arab country. Clinicians and public health policy makers can translate this evidence into health policies and practices to improve the health status and services for mothers and children.

\section{Author affiliations}

${ }^{1}$ Institute of Public Health, College of Medicine and Health Sciences, United Arab Emirates University, Al Ain, United Arab Emirates

${ }^{2}$ College of Pharmacy, Al Ain University of Science and Technology, Al Ain, United Arab Emirates

${ }^{3}$ Zayed Center for Health Sciences, United Arab Emirates University, Al Ain, United Arab Emirates

${ }^{4}$ Department of Pediatrics, College of Medicine and Health Sciences, United Arab Emirates University, Al Ain, United Arab Emirates

${ }^{5}$ Obstetrics and Gynecology Department, Oasis Hospital, Al Ain, United Arab Emirates

${ }^{6}$ Obstetrics and Gynecology Department, Tawam Hospital, Al Ain, United Arab Emirates
${ }^{7}$ Women's Health Institute, Al Ain Hospital, Al Ain, United Arab Emirates

${ }^{8}$ Department of Food Science and Human Nutrition, Agricultural University of Athens, Athens, Greece

${ }^{9}$ College of Medicine, Mohammed Bin Rashid University of Medicine and Health Sciences, Dubai, United Arab Emirates

Acknowledgements The authors would like to thank all women who generously accepted to participate in this study. The authors would like to thank the participating hospitals and antenatal clinics' doctors and nurses for their facilitation of the study. The authors would also like to thank the research assistants and data collectors who are based in the institutions and assist with recruitment.

Contributors LAA, TL and IB conceived, designed and initiated the study. LAA, FA-M, HN, CB, SG-A, FMAS, AZ, TL and IB contributed to the planning of the study. LAA, AAH, NA, IE, HE, TL and IB contributed to the implementation, coordination and management of the study. LAA, AAH, NA, HE and TL drafted this manuscript. All authors read and approved the final version of the manuscript.

Funding This work was supported by grants from Zayed Center for Health Sciences, United Arab Emirates University (31R076, 31R183), College of Medicine and Health Sciences, United Arab Emirates University (31M266) and the Mohammed Bin Rashid University-Al Mahmeed Collaborative Research Award (ALM1815). The study protocol has been peer-reviewed by national and international experts before being funded.

Competing interests None daclared.

Patient consent for publication Not required.

Ethics approval The study was approved by the United Arab Emirates University Human Research Ethics Committee (previously known as Al Ain Medical District Human Research Ethics Committee) (ERH-2017-5512), Al Ain Hospital Research Ethics Committee (AAHEC-03-17-058) and Tawam Human Research Ethics Committee (T-HREC-494).

Provenance and peer review Not commissioned; externally peer reviewed.

Open access This is an open access article distributed in accordance with the Creative Commons Attribution Non Commercial (CC BY-NC 4.0) license, which permits others to distribute, remix, adapt, build upon this work non-commercially, and license their derivative works on different terms, provided the original work is properly cited, appropriate credit is given, any changes made indicated, and the use is non-commercial. See: http://creativecommons.org/licenses/by-nc/4.0/.

\section{REFERENCES}

1. Gluckman PD, Hanson MA, Cooper C, et al. Effect of in utero and early-life conditions on adult health and disease. $N$ Engl J Med 2008;359:61-73.

2. Singhal A, Lucas A. Early origins of cardiovascular disease: is there a unifying hypothesis? The Lancet 2004;363:1642-5.

3. Gillman MW. Developmental origins of health and disease. N Engl J Med 2005;353:1848-50.

4. Golding J. Who should be studied and when in a longitudinal birth cohort? Paediatr Perinat Epidemiol 2009;23 Suppl 1:15-22.

5. Christian P, Stewart CP. Maternal micronutrient deficiency, fetal development, and the risk of chronic disease. J Nutr 2010;140:437-45.

6. Greenough A. Editor prenatal factors in the development of chronic lung disease. seminars in fetal and neonatal medicine. Elsevier, 2009.

7. Singhal A. Early nutrition and long-term cardiovascular health. Nutr Rev 2006;64:44-9.

8. Jayet P-Y, Rimoldi SF, Stuber T, et al. Pulmonary and systemic vascular dysfunction in young offspring of mothers with preeclampsia. Circulation 2010;122:488-94.

9. Larsen PS, Kamper-Jørgensen M, Adamson A, et al. Pregnancy and birth cohort resources in Europe: a large opportunity for aetiological child health research. Paediatr Perinat Epidemiol 2013;27:393-414.

10. Statistics Center - Abu Dhabi. Statistical Yearbook of Abu Dhabi 2017. Population and demography, Pages 111-156.. Available: https://www.scad.ae/Release\%20Documents/Statistical\% 20Yearbook\%20of\%20Abu\%20Dhabi-2017-EN-1May\%2018.PDF [Accessed 30 May 2018].

11. Health Authority of Abu Dhabi. HAAD standard for routine antenatal screening and care, 2012. Available: https://www.haad.ae/HAAD/ LinkClick.aspx?fileticket=NGHLA-XduVg\%3D\&tabid=820 [Accessed 30 May 2018]. 
12. Fraser A, Macdonald-Wallis C, Tilling K, et al. Cohort profile: the Avon longitudinal study of parents and children: ALSPAC mothers cohort. Int J Epidemiol 2013;42:97-110.

13. Magnus $\mathrm{P}$, Irgens LM, Haug K, et al. Cohort profile: the Norwegian mother and child cohort study (MobA). Int J Epidemiol 2006;35:1146-50.

14. Magnus $\mathrm{P}$, Birke $\mathrm{C}$, Vejrup $\mathrm{K}$, et al. Cohort profile update: the Norwegian mother and child cohort study (MobA). Int J Epidemiol 2016;45:382-8.

15. Olsen J, Melbye M, Olsen SF, et al. The Danish National Birth Cohort - its background, structure and aim. Scand J Public Health 2001;29:300-7.

16. Raynor P, Born in Bradford Collaborative Group. Born in Bradford, a cohort study of babies born in Bradford, and their parents: protocol for the recruitment phase. BMC Public Health 2008;8:327.

17. Dehghan M, Al Hamad N, Yusufali A, et al. Development of a semiquantitative food frequency questionnaire for use in United Arab Emirates and Kuwait based on local foods. Nutr J 2005;4:18.

18. Bergink V, Kooistra L, Lambregtse-van den Berg MP, et al. Validation of the Edinburgh depression scale during pregnancy. J Psychosom Res 2011;70:385-9.

19. Spielberger CD. State-Trait anxiety inventory. Wiley online library, 2010.

20. Bernstein DP, Stein JA, Newcomb MD, et al. Development and validation of a brief screening version of the childhood trauma questionnaire. Child Abuse Negl 2003;27:169-90.

21. Dube SR, Anda RF, Felitti VJ, et al. Childhood abuse, household dysfunction, and the risk of attempted suicide throughout the life span: findings from the adverse childhood experiences study. JAMA 2001;286:3089-96.

22. Rockett HRH, Wolf AM, Colditz GA. Development and reproducibility of a food frequency questionnaire to assess diets of older children and adolescents. J Am Diet Assoc 1995;95:336-40.

23. Janz KF, Lutuchy EM, Wenthe $P$, et al. Measuring activity in children and adolescents using self-report: PAQ-C and PAQ-A. Med Sci Sports Exerc 2008;40:767-72.

24. Miller CJ, Dunn EV, Berg B, et al. A hematological survey of preschool children of the United Arab Emirates. Saudi Med $J$ 2003;24:609-13.

25. Radwan H, Hashim M, Shaker Obaid R, et al. The mother-infant study cohort (MISC): methodology, challenges, and baseline characteristics. PLoS One 2018;13:e0198278.

26. Rashad H, Osman M, Roudi-Fahimi F. Marriage in the Arab world: population reference bureau (PRB) Washington 2005.

27. Razzak H, El-Metwally A, Harbi A, et al. The prevalence and risk factors of obesity in the United Arab Emirates. Saudi Journal of Obesity 2017:5:57-65.

28. AlBlooshi A, Shaban S, AlTunaiji M, et al. Increasing obesity rates in school children in United Arab Emirates. Obes Sci Pract 2016;2:196-202.

29. Okunoye G, Konje J, Lindow S, et al. Gestational diabetes in the Gulf region: streamlining care to optimise outcome. Journal of Local and Global Health Science 2015;2015.

30. Al-Houqani M, Ali R, Hajat C. Tobacco smoking using Midwakh is an emerging health problem - evidence from a large cross-sectional survey in the United Arab Emirates. PLoS One 2012;7:e39189. 